\title{
Programmes de sélection du bovin N'Dama en Afrique de l'Ouest: cas du Sénégal, du Mali et de la Gambie
}

\author{
Younouss Camara ${ }^{1,2, *}$, Mouhamadou Moustapha Sissokho ${ }^{2}$, Moussa Sall ${ }^{2}$, Frédéric Farnir ${ }^{1,3}$ \\ et Nicolas Antoine-Moussiaux ${ }^{1,3}$ \\ ${ }^{1}$ Fundamental and Applied Research for Animals \& Health (FARAH), Sustainable Animal Production, Faculté de médecine vétérinaire, \\ Université de Liège, 4000 Liège, Belgique \\ ${ }^{2}$ Centre de recherches zootechniques de Kolda, Institut sénégalais de recherches agricoles (ISRA), Bel Air, Route des hydrocarbures, BP \\ 3120 Dakar, Sénégal \\ ${ }^{3}$ Institut vétérinaire tropical, Faculté de médecine vétérinaire, Université de Liège, 4000 Liège, Belgique
}

\begin{abstract}
Résumé - La sélection génétique est un moyen pour augmenter la productivité des races endémiques dans des environnements hostiles. La trypanotolérance du bovin N'Dama a été l'argument central de la mise en œuvre des programmes de sélection de cette race en Gambie, au Mali et au Sénégal. Ces programmes ont eu des trajectoires différentes selon les objectifs, le processus de conception et les stratégies développées. L'analyse de ces trajectoires aiderait à mieux comprendre leurs succès et leurs échecs. Pour ce faire, une étude comparative de ces programmes a été réalisée en se basant sur une littérature scientifique publiée et non publiée et des entretiens avec les acteurs. L'analyse a d'abord consisté à décrire chaque programme. Une discussion a ensuite été menée pour évaluer les facteurs de succès et d'échec. Cette analyse a montré que ces facteurs dépendent de l'intérêt des éleveurs en lien avec leurs objectifs de production et leurs pratiques, de la stratégie utilisée tenant compte de l'intégration ou non de la trypanotolérance dans les critères de sélection, et de la mobilisation des ressources financières et humaines. Ces programmes de sélection ont aussi généré un capital d'expériences, dont la valorisation permettrait d'améliorer les systèmes actuels et de bâtir des programmes durables de sélection pour les systèmes d'élevage à faibles niveaux d'intrants dans les pays en développement.
\end{abstract}

Mots clés : amélioration génétique / taurin N’Dama / Gambie / Mali / Sénégal

\begin{abstract}
N'Dama cattle breeding programs in West Africa: case of Senegal, Mali and The Gambia. Genetic selection is a way to increase the productivity of endemic breeds in hostile environments. Trypanotolerance of N'Dama cattle has been the central argument for the implementation of breeding programs for this breed in Gambia, Mali and Senegal. These programs have had different trajectories depending on the objectives, the design process and the strategies developed. The analysis of these trajectories would help to better understand their successes and failures. To this end, a comparative study of these programs is carried out based on published and unpublished scientific literature and interviews with stakeholders. The analysis first consisted in describing each program. A discussion is then held to assess the success and failure factors. This analysis showed that these factors depend on the interest of farmers in relation to their production objectives and practices, the strategy used, whether or not trypanotolerance is included in the selection criteria, and the mobilization of financial and human resources. These breeding programs have also generated a wealth of experience that could be used to improve current systems and build sustainable breeding programs for low-input livestock systems in developing countries.
\end{abstract}

Keywords: genetic improvement / N'Dama cattle / Gambia / Mali / Senegal

\footnotetext{
*Auteur de correspondance : younouss.camara@isra.sn
} 


\section{Introduction}

Le taurin N'Dama est élevé en Afrique de l'Ouest pour valoriser de vastes étendues de territoires de savanes humides et infestées par des glossines, vecteurs de la trypanosomose. La race présente en effet une adaptation à ces conditions écologiques, et particulièrement une aptitude à travailler et à produire malgré l'infestation par le trypanosome, dénommée trypanotolérance (Naessens, 2006). Par conséquent, les difficultés de la lutte contre la trypanosomose et les pertes occasionnées par cette maladie (500 millions de dollars US par année à l'échelle sous-régionale) font de cet animal une race de choix pour les petits éleveurs des écosystèmes humides (Ehui et al., 2003). Toutefois, en dépit de cette remarquable adaptation, le bovin N'Dama ne satisfait pas les objectifs de production laitière des éleveurs des pays concernés. Ses performances de production sont faibles, avec $0,66 \mathrm{~kg}$ de lait par jour, soit $80 \mathrm{~kg}$ par lactation d'une durée moyenne de 4 mois et un rendement moyen d'abattage de $145 \mathrm{~kg}$ de viande par animal (Dieye et al., 2002). Aussi, l'une des stratégies de ces pays a été la mise en place de programmes d'amélioration génétique des performances de production du bovin N'Dama.

Plusieurs programmes de sélection des animaux de race locale ont été initiés (Fall et al., 1982; Planchenault et al., 1984 ; Dempfle et Jaitner, 2000). Ceux concernant la race N'Dama ont été développés au Sénégal en 1972 (Fall et al., 1982), au Mali en 1975 (Tamboura et al., 1982), et en Gambie en 1994 (Bosso et al., 2007). Une étude comparative de ces programmes présente plusieurs intérêts :

- les trois programmes concernent la même race;

- ils ont débuté au Sénégal et au Mali à la même période (années 1970);

- ils partagent les mêmes réalités environnementales et socio-culturelles;

- ils ont fait l'objet d'un appui en 2008 de la Banque africaine de développement (BAD) et de l'Organisation des Nations unies pour l'alimentation et l'agriculture (FAO), dans le cadre du Projet de gestion durable du bétail ruminant endémique (PROGEBE).

Plusieurs études se sont intéressées à l'évaluation de ces programmes à travers une analyse zootechnique ou génétique (Fall et al., 1982; Planchenault et al., 1984; Bosso et al., 2009; Camara, 2012). Cependant, seul le cas de la Gambie a fait l'objet d'une évaluation centrée sur les stratégies de sélection mises en œuvre (Bosso et al., 2007).

Dans cette étude, nous entendons par stratégies toutes les actions développées. Elles concernent le mode de financement, la définition des objectifs et des critères de sélection, les schémas adoptés, l'implication des parties prenantes et leur interaction dans la mise en œuvre de ces programmes. Dès lors, l'hypothèse de cette recherche est que le succès de ces programmes dépend de ces stratégies et de leur appropriation par les éleveurs.

L'objectif de cette étude est de faire une revue critique de ces programmes de sélection en mettant en évidence les manquements des stratégies mises en œuvre ainsi que leurs facteurs de succès ou d'échec. Cela permettra d'identifier des opportunités d'amélioration des schémas actuels et par ailleurs de comprendre les déterminants du succès des programmes de sélection des systèmes à faible niveau d'intrants des pays en développement.

\section{Matériel et méthode}

\subsection{Collecte des informations}

Cette étude se base sur une analyse bibliographique de la littérature scientifique publiée (articles scientifiques, livres et chapitres de livre) et non publiée ou grise (fiches techniques, mémoires et thèses), et de l'exploitation de tout autre document réalisé dans le cadre de ces programmes (rapports d'activités de recherche et de projets, comptes-rendus de mission et procès-verbaux de réunions). Pour analyser la perception des acteurs sur les stratégies développées et les périodes d'inactivité des programmes, nous avons utilisé comme sources complémentaires d'information :

- des entretiens semi-directifs sur ces thématiques avec les personnes ressources (chercheurs, agents d'élevage et éleveurs);

- des observations participantes dans les réunions auxquelles nous avons participé avec des acteurs du programme de sélection.

Dans les cas du Mali et de la Gambie, ces informations ont été collectées par des échanges de courriers électroniques avec les responsables des programmes.

\subsection{Analyse des informations collectées}

L'analyse s'est focalisée sur les stratégies développées pour mener les programmes de sélection. Dans un premier temps, le programme de chaque pays est décrit séparément. Dans cette partie, la recherche a été orientée sur le contexte d'implantation, le financement, les objectifs visés, les stratégies de sélection mises en œuvre et sur l'implication des autres services techniques (services d'élevage, projets et Organisations non gouvernementales [ONG]) et des éleveurs dans le processus. Pour compenser le déséquilibre observé dans la description des programmes, nous avons axé dans un second temps la discussion générale sur l'identification des points forts et faibles des schémas exécutés et sur les opportunités pouvant contribuer à améliorer les stratégies actuelles. Cette partie s'est basée sur une analyse thématique des mots-clés soulevés dans le discours des acteurs, issus des notes prises lors des entretiens semi-directifs (Camara et al., 2019a).

\section{Amélioration génétique du bovin N'Dama au Sénégal}

\subsection{Objectifs du programme}

Mis en place en 1972 dans la zone subhumide au sud du pays avec le soutien financier du Fonds africain de coopération (FAC) et de l'État sénégalais, le programme de sélection du bovin N'Dama au Sénégal avait pour objectif de départ d'améliorer la qualité bouchère de la race (Fall et al., 1982). Cet objectif initial, défini par l'Institut sénégalais de recherches agricoles (ISRA), initiateur du programme via son Centre de 
recherche zootechnique (CRZ) de Kolda sur la base de la littérature alors disponible, s'est vite montré en inadéquation avec ceux des éleveurs, pour lesquels le lait représente une valeur alimentaire, monétaire et culturelle majeure. À partir de 1990, la prise en compte de la production laitière dans les critères de sélection a eu pour objectif, d'une part, d'avoir une estimation des performances laitières, et d'autre part, de se rapprocher des pratiques d'élevage en vigueur en milieu traditionnel. Avant cette date, aucune enquête participative n'avait été faite pour évaluer les objectifs d'élevage des éleveurs. Toutefois, les faibles performances laitières de cette race étaient un indice suffisant pour les responsables du programme pour sélectionner le bovin N'Dama sur ses performances bouchères. En 1991, dans le but de faire contribuer les éleveurs à l'effort de sélection (les caractéristiques des éleveurs participants ont été décrites par Camara et al., 2019b) et d'intégrer leurs objectifs, le programme a adopté un nouveau système dénommé SAGNO (Système d'amélioration génétique à noyau ouvert) et a affiché l'amélioration des capacités laitières et de trypanotolérance parmi ses objectifs (Diop et al., 1993).

\subsection{Schémas de sélection}

Deux schémas ont été expérimentés selon les difficultés et les opportunités rencontrées. Tout d'abord, un schéma à noyau fermé a été expérimenté, qui reposait sur un troupeau de sélection d'environ 400 têtes et avec un taux de consanguinité $\Delta \mathrm{F}$ de départ de 0,016 . Le système fonctionnait sur deux niveaux: le noyau de sélection, où le progrès génétique est produit, et les troupeaux des éleveurs, où il est diffusé (Fall, 1989). Des limites ont été identifiées dès 1987 quant à son impact sur la productivité des troupeaux villageois. Elles concernaient l'absence de la production laitière dans les objectifs de sélection, la non-implication des éleveurs et la taille restreinte du noyau (Diop et al., 1993). Sur ce diagnostic, un système à noyau ouvert a été adopté au début des années 1990. Ce système comportait deux variantes selon le niveau d'organisation et d'implication des éleveurs et le nombre d'étages composant le schéma.

De 1991 à 1996, un système à noyau ouvert à deux étages a été appliqué avec l'appui de l'Agence des États-Unis pour le développement international (USAID). Il reposait sur trois étapes essentielles: 1) le dépistage des futures mères à taureaux dans les troupeaux villageois ; 2) le testage en station d'un noyau ; 3) l'utilisation des taureaux sélectionnés comme géniteurs chez les éleveurs (Diop et al., 1993). Si les étapes 1 et 3 étaient conjointement menées par le CRZ et les éleveurs, l'étape 2 était exclusivement réservée au CRZ.

À la fin de l'appui de l'USAID, le programme est entré en période de léthargie entre 1996 et 2008, essentiellement à cause d'un défaut de financement (Sissokho et al., 1993). Les troupeaux étaient maintenus sans aucune opération de sélection (contrôle de performance, testage et diffusion de géniteurs). Toutefois, selon ces auteurs, une gestion conservatoire (enregistrement des naissances, des mortalités et des pedigrees, conduite aux pâturages et gestion sanitaire) était appliquée dans le noyau de sélection.

À sa reprise en 2008, avec le financement de la BAD et de la FAO dans le cadre du PROGEBE, le programme de sélection a adopté un système de sélection à noyau ouvert à trois étages : le noyau de sélection où le progrès génétique est produit, le troupeau de multiplication détenu par les éleveurs de la coopérative des agroéleveurs sélectionneurs du bovin N'Dama (CASE N'Dama, créée pour améliorer la gouvernance du programme de sélection) et les troupeaux commerciaux (ou villageois) pour le diffuser. Un des objectifs opérationnels du schéma était de maintenir la taille du noyau de sélection à 200 femelles avec 4 mâles pour un $\Delta \mathrm{F}$ égal à 0,039 . Ce système autorise l'introduction et le testage dans le noyau de jeunes taurillons (12 à 24 mois) issus des villages. Contrairement aux schémas précédents, les éleveurs interviennent à toutes les étapes du processus de sélection (noyau de sélection, troupeaux de multiplication, diffusion du progrès génétique et troupeau villageois).

\subsection{Processus et critères de sélection}

La sélection des mères à taureaux se fait en trois étapes. La première sélection est faite par les éleveurs qui identifient les meilleures vaches en se basant sur leur carrière de production laitière. Ensuite, un contrôle laitier est effectué sur ces vaches et enfin une analyse statistique.

Les animaux sont alors transférés dans le noyau de sélection pour le testage. Les critères de sélection du premier schéma portaient sur le gain quotidien moyen (GQM) de 6 à 18 mois et de 18 à 24 mois, sur la qualité spermatique et sur la conformation (Fall et al., 1982). Cependant, la couleur fauve de la robe, présumée être un indice de trypanotolérance (Touré, 1977), était aussi prise en compte.

Dans le schéma ouvert, la sélection finale se fait à 36 mois et passe par une phase de présélection et de sélection. La présélection concerne les veaux mâles de 6 à 18 mois, le critère de sélection étant le poids à âge type, supérieur à $150 \mathrm{~kg}$ à 18 mois. Le testage en enclos individuel suit alors le GQM de 18 à 36 mois. La prise en compte de l'hématocrite dans l'index de sélection a été envisagée lors de ce schéma. Il n'a pas été utilisé de façon pratique car la corrélation positive variant de 0,40 à 0,70 entre l'hématocrite et la croissance permettrait d'en tenir compte indirectement (Abenga et Vuza, 2005). Jusque dans les années 1990, le classement final des animaux s'est fait selon une analyse statistique utilisant la méthode des moindres carrés. Cependant, en 2012, une analyse génétique utilisant la méthode BLUP (Best Linear Unbiaised Prediction) a montré un gain génétique annuel de $0,430 \mathrm{~kg}$ pour le poids adulte à 36 mois et une héritabilité estimée variant de 0,07 à 0,12 (pour les poids à la naissance et à 24 mois respectivement) (Camara, 2012).

\subsection{Implication des éleveurs dans le programme}

Jusqu'en 1990, la diffusion des géniteurs dans les troupeaux s'est faite par vente. Durant la phase test d'ouverture du noyau entre 1991 et 1996, les éleveurs ont bénéficié d'échanges de géniteurs contre l'introduction de leurs vaches dans le noyau. Depuis 2008, la création d'une coopérative d'agroéleveurs sélectionneurs de la N'Dama (CASE N'Dama) coordonne la multiplication des géniteurs sélectionnés et la diffusion de leurs gènes. Elle contribue également à la gestion du noyau de sélection. La CASE N'Dama est chargée de 
Tableau 1. Diffusion du progrès génétique du noyau de sélection vers les troupeaux villageois de 2011 à 2016 au Sénégal. Table 1. Diffusion of genetic progress from the breeding nucleus to village herds from 2011 to 2016 in Senegal.

\begin{tabular}{lllll}
\hline Troupeaux & $\begin{array}{l}\text { Troupeaux } \\
\text { ciblés }(n)\end{array}$ & $\begin{array}{c}\text { Troupeaux } \\
\text { atteints }(n)\end{array}$ & $\begin{array}{c}\text { Géniteurs du noyau } \\
\text { vers la multiplication }(n)\end{array}$ & $\begin{array}{c}\text { Géniteurs de la multiplication } \\
\text { vers les troupeaux villageois }(n)\end{array}$ \\
\hline Multiplication & 30 & 21 & 21 & 42 \\
Villages & - & 41 & 05 & 42 \\
Total & 30 & 62 & 26 & \\
\hline
\end{tabular}

$n=$ nombre.

sensibiliser et d'informer les éleveurs sur la disponibilité des géniteurs. Elle identifie les éleveurs demandeurs et, sur la base d'un protocole qui la lie au CRZ, donne son avis sur l'animal sélectionné à diffuser. Cette collaboration avec les éleveurs a permis la diffusion de 21 géniteurs dans les troupeaux de multiplication et de 47 géniteurs de seconde génération dans les troupeaux villageois (Tab. 1).

\section{Amélioration génétique du bovin N'Dama au Mali}

\subsection{Objectifs du programme}

$\mathrm{Au}$ Mali, les programmes d'amélioration génétique ont commencé dès 1960 au Centre national de recherche zootechnique de Sotuba (Tamboura et al., 1982) par le croisement de la N'Dama avec les races européennes et le zébu Peul du Mali. L'objectif était de tirer parti de la trypanotolérance de la race N'Dama et de la productivité du zébu Peul et des races européennes. Le bovin N'Dama fera également l'objet d'un programme de sélection en race pure dans le cadre de l'opération N'Dama de Yanfolila (ONDY), mise en place en 1975, dont l'objectif était de créer un berceau pour la race N'Dama et d'améliorer ses caractéristiques bouchères (Tamboura et al., 1982).

\subsection{Schéma de sélection}

Quatre phases ont été observées. Entre 1975 et 1981, un ranch de 10000 ha et un troupeau de sélection de 1800 animaux de race N'Dama ont été mis en place. Entre 1981 et 1986, une méthode de sélection de type massale a été mise au point et développée, avec comme objectif la création d'une souche de race N'Dama dans le cercle de Madina Diassa (Sikasso, Mali), qui servirait de base de référence à la sélection (Planchenault et al., 1984). Le noyau de sélection était donc fermé à tout apport génétique externe pouvant compromettre cet objectif. Cependant, les carences constatées dans l'alimentation et la conduite du troupeau ont empêché une bonne extériorisation du potentiel génétique des animaux et les coûts de la gestion du ranch, jugés élevés, se sont ajoutés à ces contraintes (Berti et al., 1995). Les difficultés apparues lors de la deuxième phase ont donc entraîné une réorientation vers une gestion participative du programme par le Service de l'élevage du Mali, afin d'y associer les éleveurs.

Cette gestion participative s'est matérialisée entre 1991 et 1993 par le transfert des bovins sélectionnés chez les éleveurs. L'objectif était aussi de vérifier l'adaptabilité de la sélection au système d'élevage des éleveurs, de consolider la sélection, d'adapter la méthode et de mettre en œuvre une méthode de gestion pastorale tenant compte des pratiques villageoises et des objectifs des éleveurs, devant résoudre le problème de l'alimentation (Berti et al., 1995). La méthode de diffusion utilisée lors de cette phase était basée sur le principe du contrat de prêt bétail, établi sur 10 ans et prévoyant le remboursement, nombre par nombre et sexe par sexe, du bétail prêté (Berti et al., 1995). Cette diffusion a été menée dans le cadre du programme d'appui aux éleveurs, à travers une ligne de crédit située à la Banque nationale de développement agricole (BNDA).

Cette opération de transfert a permis d'étendre la base de sélection aux troupeaux villageois sous forme de schéma de sélection à plusieurs noyaux en milieu ouvert. Mais le programme n'a pas pu se poursuivre à cause d'un déficit de financement. Il a été repris en 2008, avec l'appui financier du PROGEBE, sous la forme d'un schéma de sélection à noyau central ouvert aux animaux des éleveurs. Les prêts bétail développés lors de la troisième phase ont permis de reconstituer le noyau de sélection et la création du Centre de conservation et de multiplication du bétail ruminant endémique de Médina Diassa (CCMD/BRE) (Coulibaly et Diallo, 2014).

\subsection{Critères de sélection}

La sélection a été faite en prenant comme référence un animal de type fauve sans tache ou de type froment et ayant une bonne conformation (massive et trapue) (Planchenault et al., 1984). Les animaux répondant à ces critères ont été choisis sur la base de leur GQM entre 8 et 18 mois et le poids à âge type (PAT), supérieur ou égal à $150 \mathrm{~kg}$ à 18 mois. Lors des dépistages, les critères de sélection consistaient en une mesure du périmètre thoracique. La trypanotolérance était incluse dans les critères de sélection en utilisant faiblement les trypanocides, qui étaient uniquement réservés aux cas sévères de trypanosomose. L'évaluation faite par Planchenault et al. (1984) a indiqué une évolution de la croissance des poids des animaux sélectionnés de la naissance au sevrage, avec un GQM de $239 \mathrm{~g} / \mathrm{j}$ contre $222 \mathrm{~g} / \mathrm{j}$ pour les animaux élevés chez les éleveurs.

\subsection{Implication des éleveurs dans le programme}

Le transfert des animaux chez les éleveurs a permis de toucher un effectif de près de 3000 têtes. Cependant, les 
Tableau 2. Développement chronologique du programme d'amélioration génétique des bovins N'Dama en Gambie (Bosso et al., 2007). Table 2. Chronological development of the N'Dama cattle genetic improvement programme in The Gambia (Bosso et al., 2007).

\begin{tabular}{ll}
\hline Évènements & Période \\
\hline Création de l'ITC & 1984 \\
Mission de consultation de l'Organisation des Nations unies pour l'alimentation et l'agriculture (FAO) & \\
effectuée à l'ITC & 1990 \\
Nécessité exprimée d'un programme d'amélioration cohérent et complet & 1993 \\
Proposition d'un schéma de sélection à noyau ouvert & 1993 \\
Financement du schéma de sélection à noyau ouvert par la coopération allemande & 1993 \\
(Bundesministerium für Wirtschaftliche Zusammenarbeit, BMZ) & 1994 \\
Lancement du programme & 1995 \\
Début de l'enregistrement des performances & 1996 \\
Atelier organisé pour définir les objectifs de sélection & $1996 / 1999$ \\
Achat d'animaux de remplacement (dépistage) & 1998 \\
Accord sur les objectifs de sélection & 1998 \\
Introduction d'une évaluation BLUP de modèle animal & 2001 \\
Introduction d'un mécanisme de diffusion du progrès génétique & 2000 \\
Financement du Projet PROCORDEL pour la diffusion des géniteurs améliorés \\
Financement OPEP-FID pour des opérations de dépistage \\
Création d'associations d'éleveurs pour la multiplication du bétail (Gambian Indigenous Livestock Multiplier \\
Association, GILMA) \\
Enquête sur l'avis des agriculteurs sur le programme de sélection \\
Financement du PROGEBE \\
\hline
\end{tabular}

rapports consultés indiquent une interaction limitée entre les agents d'élevage, les décideurs économiques et les éleveurs. En effet, d'après ces rapports, le transfert des animaux chez les éleveurs n'a pas été accompagné d'un suivi zoosanitaire de la part des agents des services d'élevage pour contrôler les performances de production. En outre, les éleveurs n'étaient pas organisés dans le cadre d'une association pouvant interagir avec ces acteurs de l'élevage. Ce cadre jouerait un rôle important dans la diffusion des géniteurs améliorés, comme observé au Sénégal. Toutefois, les éleveurs sélectionneurs ayant bénéficié des animaux lors de la troisième phase continuent aujourd'hui de sélectionner et de vendre la souche N'Dama de Madina Diassa.

\section{Amélioration génétique du bovin N'Dama en Gambie}

\subsection{Objectifs du programme}

Le programme de sélection du bovin N'Dama en Gambie a débuté en 1994 dans un contexte de pression glossinaire importante, d'une forte demande en lait et en viande et de l'amélioration générale de la sécurité alimentaire (Dempfle, 1993). En 1993, la nécessité d'un programme cohérent et complet d'amélioration génétique avait été exprimée (Bosso et al., 2007). Le tableau 2 donne la chronologie des différents évènements du programme.

Les objectifs du programme, consistant à accroître les productions de viande et de lait sans compromettre les capacités d'adaptation et de résistance aux maladies de la race, ont été définis dès le départ de manière participative entre le Service national de recherche agricole (NARS pour National Agriculture Research Service) et les éleveurs (Bosso et al., 2007).

\subsection{Schéma de sélection}

La stratégie était basée sur un système de sélection à noyau ouvert à trois niveaux, incluant le noyau (production du progrès génétique, niveau géré par la recherche), la multiplication (troupeaux qualifiés au niveau communautaire, niveau géré par les éleveurs) et les élevages commerciaux (diffusion du progrès génétique). La consanguinité était gérée dans le noyau, par l'introduction des descendants mâles des meilleures vaches des fermes participantes (Jaitner et al., 2003).

Le noyau de sélection était géré dans deux stations: Kenaba et Bansang. La première était caractérisée par une pression glossinaire moyenne à faible. Elle mettait en œuvre la reproduction et le testage des jeunes taureaux jusqu'au sevrage à 12 mois. La seconde était caractérisée par une forte pression glossinaire. Elle mettait en œuvre la sélection finale à 36 mois. Les meilleurs animaux démontrant leur tolérance dans ces conditions d'élevage étaient choisis et retournés à Kenaba pour la reproduction.

\subsection{Critères de sélection}

Les animaux étaient sélectionnés selon un index composite, déterminé de façon participative par les éleveurs et les chercheurs du NARS, et contenant des informations sur les performances de croissance des jeunes taureaux et des apparentés: le GQM de 0 à 12 mois en zone contrôlée à Kenaba, le GQM de 15 à 36 mois en zone difficile à faible niveau d'intrants à Bansang et la production laitière sur les 100 premiers jours de lactation des mères à taureaux (Bosso et al., 2007). Le critère de trypanotolérance, mais aussi d'adaptation plus générale aux conditions d'élevage prévalant dans la région, était ainsi pris en compte. Une évaluation 
génétique réalisée selon le modèle BLUP a indiqué un gain génétique de $0,40 \mathrm{~kg}$ par an et une héritabilité estimée variant de 0,28 à 0,48 (pour les poids à 36 et 15 mois respectivement) (Bosso et al., 2007).

\subsection{Implication des éleveurs dans le programme}

Les éleveurs, pratiquant l'agropastoralisme et organisés au sein d'une association de sélectionneurs appelée «Association des multiplicateurs de bétail indigène de Gambie» (GILMA, pour Gambian Indigenous Livestock Multiplier Association) par le Centre international de trypanotolérance (ITC, pour International Trypanotolerance Center), ont joué un grand rôle dans la diffusion du progrès génétique du programme (Bosso et al., 2007). Les agriculteurs devenaient membres de l'association s'ils recevaient un animal reproducteur de l'ITC et acceptaient de respecter les règles de l'association.

Le rôle de la GILMA dans le programme de sélection était de coordonner la multiplication des géniteurs issus du noyau de sélection et de diffuser leur progéniture chez les éleveurs. Pour mieux assurer le rôle des différentes parties prenantes, les capacités institutionnelles de gouvernance du programme de sélection ont été renforcées et une politique d'incitations (subventions accordées aux éleveurs pour une meilleure participation) a été mise en place (Bosso et al., 2007).

\section{Discussion}

Les cas exposés ici montrent l'importance pour la pérennisation des programmes de sélection de l'adéquation entre leurs objectifs de sélection et ceux des éleveurs, l'implication de toutes les parties prenantes, leur interaction, la gouvernance et les modes de financement des programmes.

\subsection{Objectifs de sélection}

La prise en compte des objectifs des éleveurs a été à l'origine des schémas développés et de la réorientation des programmes au Sénégal et en Gambie (Ejlertsen et al., 2013 ; ILRI, 2010). En effet, l'intérêt des éleveurs pour la production laitière et sa prise en compte dans les critères de sélection ont été à l'origine du changement opéré par le programme du Sénégal. L'intérêt des éleveurs pour cet objectif de production a également été confirmé par la méthode participative mobilisée dans l'identification des objectifs de sélection en Gambie. Dans ce pays, les éleveurs ont été associés dès le départ à la conception du projet en tenant compte de leurs objectifs de production (Dempfle et Jaitner, 2000). Alors qu'au Sénégal, la conception du premier schéma reposait sur des objectifs établis par les chercheurs, amenant le programme à se réorienter face à la faible adhésion des éleveurs. Le bovin N'Dama n'étant pas une race laitière, cela expliquerait les difficultés pratiques et techniques pour prendre en compte des critères basés sur le lait. Cet objectif de production motiverait les éleveurs de bovins N'Dama au Mali et au Sénégal à les croiser avec des races laitières ou avec des zébus, montrant ainsi l'inadéquation des programmes avec cette pratique (Traoré et al., 2018; Camara et al., 2019b).
Dans le cas du Sénégal, on peut en outre remarquer que si l'amélioration du format de l'animal a été retenue dès le départ comme objectif de sélection, l'activité effective de sélection mêlait la performance bouchère à un objectif de trypanotolérance, s'opérationnalisant à travers une standardisation de la robe des animaux (Touré, 1977). Ce même critère avait prévalu au Mali, dont l'objectif était de sélectionner une souche N'Dama de robe fauve ou froment de Madina Diassa (Planchenault et al., 1984). Aucun de ces deux programmes ne peut donc être considéré comme ayant poursuivi de manière stricte un travail de sélection pour l'accroissement de la productivité individuelle, sans pour autant avoir inclus de manière rigoureuse de critère de trypanotolérance ou d'adaptation aux conditions d'élevage à faibles niveaux d'intrant. En effet, l'adaptation des races indigènes, impliquant leur adoption par les éleveurs car répondant à leur système d'élevage pour des objectifs de production bien définis (Agabriel et al., 2014; Couix et al., 2016), doit être incluse rigoureusement dans l'index de sélection. Cela constitue une condition de durabilité des activités d'élevage.

\subsection{Schémas de sélection}

Dans les trois programmes, les caractéristiques de trypanotolérance du bovin N'Dama ont été retenues dès le départ. Comme souligné précédemment, la difficulté reposait sur son intégration dans un index de sélection. Cette principale contrainte, associée à d'autres (insuffisance des ressources humaines, faiblesse de financement ou défaut d'infrastructures), a défini les schémas retenus. Avec l'intégration des éleveurs dans le processus de sélection, les schémas sénégalais et gambiens reposaient sur un système à trois étages. Le cas du Mali n'était quant à lui pas spécifié, l'étape de multiplication du progrès génétique n'ayant pas été formellement décrite (Diop et al., 1993; Berti et al., 1995 ; Bosso et al., 2007).

Au Mali et au Sénégal, les schémas étaient basés au départ sur un noyau centralisé fermé à tout apport de gènes extérieurs. Le but affirmé pour le Mali était de ne pas compromettre la sélection d'une souche N'Dama typique de Madina Diassa, en ligne avec l'objectif de « création d'un berceau de la race». De manière marquante, le programme initial du Mali ne comportait pas de transfert de génétique du noyau vers les éleveurs. Malgré son affichage en tant que programme de sélection, le programme malien pouvait donc être assimilé davantage à un programme de création et de conservation d'une souche de N'Dama. Le programme du Sénégal, lui, correspondait bien dès son démarrage à un programme d'amélioration génétique, puisque les éleveurs bénéficiaient du progrès génétique produit. Les cas du Sénégal et du Mali divergent également dans les redirections de programme suite au constat d'échec des premières phases. Le Sénégal a adopté un schéma de sélection à noyau central et ouvert aux éleveurs, alors qu'au Mali, les animaux du noyau de sélection ont été transférés chez les éleveurs, créant ainsi plusieurs noyaux de sélection décentralisés.

À la différence des stratégies mises en place au Sénégal et au Mali, où les animaux étaient élevés du vêlage à la sélection finale dans le même environnement, en Gambie, le schéma à noyau ouvert a été aussi déterminé par l'intégration de la trypanotolérance comme critère de sélection, en élevant les 
animaux dans deux environnements différents. Les conditions favorables du premier noyau permettaient un meilleur taux de naissance et de sevrage et les conditions difficiles du deuxième noyau permettaient de tester les futurs reproducteurs sous des contraintes environnementales proches du milieu d'élevage réel. Toutefois, de manière plus générale, les rapports consultés ont relevé une absence de schéma de sélection structuré, qui aurait intégré les aspects de création du progrès génétique et de sa diffusion. Les centres de sélection ont souvent travaillé à la production d'animaux améliorés, mais la diffusion de ces animaux n'a pas reçu de leur part l'importance qui aurait dû lui être accordée, en partie à cause de la faible implication de toutes les parties prenantes. Au Sénégal et en Gambie, cette dernière activité est actuellement déléguée aux associations d'éleveurs créées à cet effet.

\subsection{Implication des acteurs}

L'un des critères qui a déterminé les stratégies des programmes est la prise en compte de l'intérêt des éleveurs et leur implication. En effet, les programmes d'élevage dans ces pays en développement étaient essentiellement pilotés par les services gouvernementaux, avec une très faible implication des éleveurs. Pour les cas étudiés, les formes de cette implication ont été différentes d'un programme à un autre. Selon les pays, les éleveurs sont intervenus à différents niveaux du processus de sélection. En Gambie, les éleveurs ont participé à la définition des objectifs de sélection, à la multiplication et à la diffusion du progrès génétique (Dempfle et Jaitner, 1999). Au Sénégal, en plus de leur rôle dans la multiplication et la diffusion du progrès génétique, les éleveurs sont intervenus dans le repeuplement du noyau de sélection et aussi dans le choix final des femelles futures mères à taureaux. La différence entre ces deux interventions dérive d'une vision différente des concepteurs des deux programmes. Pour les responsables du programme du Sénégal, la sélection animale est un travail d'éleveurs. Dès lors, ces derniers doivent être au cœur du processus en participant à l'effort de sélection. Cet engagement des éleveurs s'est traduit par le renforcement du noyau de sélection par une partie de leurs troupeaux. Ainsi, le noyau de sélection du Sénégal, de 2008 à nos jours, s'est composé des animaux des éleveurs et de ceux du CRZ, alors que celui de la Gambie a été entièrement constitué par des animaux appartenant à la station de recherche. L'objectif de cette implication active observée au Sénégal était de partager les charges de gestion entre les parties prenantes. Toutefois, cette approche a été source de conflits entre les parties prenantes, car certains éleveurs demandaient une compensation financière ou en nature (suivi sanitaire de leurs troupeaux ou dotation en médicaments) en contrepartie de l'introduction de leurs animaux dans le noyau de sélection. Cela aurait entraîné la démission des éleveurs du programme du Sénégal (Camara et al., 2019a). Par ailleurs, les charges non partagées peuvent aussi entraîner l'échec ou l'arrêt des programmes après le départ des bailleurs qui les finançaient, comme observé au Mali où les charges de gestion du ranch ont motivé les promoteurs à transférer les animaux chez les éleveurs. Ce modèle de gestion des noyaux de sélection décentralisés confiés aux éleveurs maliens s'apparente au modèle villageois de gestion d'un troupeau de multiplication décrit par Jaitner et al. (2003). Il pourrait aussi être considéré comme un modèle à base communautaire des programmes de sélection, proposé actuellement comme étant plus efficace pour les systèmes à faibles niveaux d'intrants (Wurzinger et al., 2013; Mueller et al., 2015; Haile et al., 2019).

L'étude comparative de ces programmes montre le rôle essentiel d'appui des bailleurs de fond dans le développement et la pérennisation des programmes de sélection dans les pays en développement (Camara et al., 2019c). L'analyse a aussi révélé que l'implication des éleveurs, à des degrés divers, à travers la mise en place d'organisations de producteurs, a créé une forte interaction entre les parties prenantes. Ces relations institutionnelles entre le CRZ et la CASE N'Dama au Sénégal, et entre l'ITC et la GILMA en Gambie, ont contribué à la production, à la multiplication et à la diffusion des géniteurs améliorés. L'analyse des cas de ces deux pays montre le rôle important d'une bonne gouvernance dans le développement des programmes de sélection à faible niveau d'intrants des pays en développement.

\subsection{Points saillants de la comparaison}

Le programme de sélection du Sénégal a généré une expérience capitale qui a été bénéfique à sa réorientation et également au développement de celui de la Gambie. Le schéma actuel repose sur un modèle de partage des charges avec les éleveurs. Celui de la Gambie avait réussi dans son approche participative en associant les éleveurs dans la définition des objectifs de sélection. Il a en outre adopté une stratégie de sélection basée sur la trypanotolérance. Au Mali, l'étendue de la base de sélection, qui aurait dû être son point fort du point de vue génétique, a été son point faible car demandant beaucoup plus de ressources financières et humaines dans la gestion du ranch. L'expérience de la création des noyaux de sélection décentralisés aurait dû être évaluée et valorisée. En effet, les modèles de communitybased breeding programs, consistant dans le développement et la gestion des programmes par les éleveurs, sont reconnus par plusieurs auteurs comme plus durables pour les systèmes à faible niveau d'intrants des pays en développement (Wurzinger et al., 2013; Mueller et al., 2015; Haile et al., 2019). Ainsi, la création des noyaux de sélection décentralisés s'apparente à ces modèles étudiés actuellement. Le prêt bétail associé, adopté comme un mode d'acquisition des animaux par les éleveurs, a permis la conservation du type N'Dama sélectionné, conduisant ainsi à la constitution du noyau de sélection central actuel. L'évaluation de ce système de prêt bétail pourrait donner des pistes pour un modèle innovant et participatif de financement des programmes de sélection dans les pays en développement, dont la pérennisation est fonction en grande partie d'une ligne de financement structurel. En effet, ces programmes, dépendant des financements externes, s'arrêtent à la fin des projets ou des programmes qui les ont mobilisés.

\section{Conclusion}

Cette étude comparative suggère que la réussite des programmes de sélection des pays étudiés dépend beaucoup des objectifs partagés avec les utilisateurs finaux et de 
l'adoption d'une stratégie basée sur l'implication des éleveurs, des financements externes et sur la prise en compte de la trypanotolérance.

Cette étude comparative apporte ainsi des éclairages uniques et complémentaires sur l'appropriation des éleveurs, les modes de financement ou l'implication et l'interaction des parties prenantes dans la gestion de ces programmes. Ces enseignements nous poussent à suggérer des pistes d'investigation pour améliorer les schémas actuels. Au cœur de ces investigations, nous soulignons en conclusion la place centrale à donner à l'analyse des arrangements institutionnels, organisant les interactions entre les acteurs, leurs rôles et responsabilités. Ces analyses sont à contextualiser dans les pays ciblés et à penser en regard des visions portées par les différentes parties prenantes ainsi que leurs capacités financières et techniques.

\section{Références}

Abenga JN, Vuza D. 2005. About factors that determine trypanotolerance and prospects for increasing resistance against trypanosomosis. African Journal of Biotechnology 4(13): 1563-1567.

Agabriel J, Faure B, Lebreton X, Garcia-launay F, Pradel P, Angeon $\mathrm{V}$, et al. 2014. La race bovine Salers: un atout pour le développement de son territoire d'origine par son identité forte et des produits qualifiés. Cahiers Agricultures 23(2): 138-147. DOI: 10.1684/agr.2014.0687.

Berti F, Renard J, Koné O. 1995. Amélioration des pratiques de l'élevage du bétail Ndama en milieu villageois: l'exemple de Madina-Diassa au Sud du Mali. Tropicultura 13(4): 164-169.

Bosso NA, Corr N, Njie M, Fall A, van der Waaij EH, van Arendonk JAM, et al. 2007. The N'Dama cattle genetic improvement programme: A review. Animal Genetic Resources Information 40: 65-69.

Bosso NA, van der Waaij EH, Kahi AK, van Arendonk JAM. 2009. Genetic analyses of N'Dama cattle breed selection schemes. Livestock Research for Rural Development 21(8): 135.

Camara Y, 2012. Analyse génétique des performances zootechniques des bovins de race N'Dama et étude du système d'amélioration génétique à noyau ouvert. Rabat (Maroc): Institut Vétérinaire et Agronomique Hassan II.

Camara Y, Ciss M, Moula N, Sissokho MM, Antoine-Moussiaux N. 2019a. Determinants of breeders' participation to an indigenous cattle breeding program. Agronomy for Sustainable Development 39(5): 44. DOI: 10.1007/s13593-019-0591-1.

Camara Y, Ciss M, Moula N, Sissokho MM, Antoine-Moussiaux N. 2019b. Analysing innovations among cattle smallholders to evaluate the adequacy of breeding programs. Animal 13(2): 417-426. DOI: 10.1017/S1751731118001544.

Camara Y, Sow F, Govoeyi B, Moula N, Sissokho MM, AntoineMoussiaux N. 2019c. Stakeholder involvement in cattle-breeding program in developing countries: A Delphi survey. Livest Sci 228: 127-135. DOI: 10.1016/j.livsci.2019.08.014.

Couix N, Gaillard C, Lauvie A, Mugnier S, Verrier E. 2016. Des races localement adaptées et adoptées, une condition de la durabilité des activités d'élevage. Cahiers Agricultures 25(6): 650009. DOI: 10.1051/cagri/2016052.

Coulibaly T, Diallo L. 2014. Diagnostic de la situation de l'élevage N'Dama dans son berceau de race (cercles de Bougouni et de Yanfolila). Bamako (Mali) : CTB, $52 \mathrm{p}$.
Dempfle L. 1993. Strategies for improving the genetic performance of local breeds. In: Proc. Atelier sur l'amélioration génétique des bovins en Afrique de l'Ouest, Banjul 17-21, pp. 2-11.

Dempfle L, Jaitner J. 1999. Handouts. In: Regional Workshop about Animal Breeding and Genetics for Applied Geneticists and Professionals Responsible for Breeding Programmes. ITC, 8-19 February 1999, unpublished, pp. 77-97.

Dempfle L, Jaitner J. 2000. Case study about the N'Dama breeding programme at the International Trypanotolerance Centre (ITC) in the Gambia. In: Galal S, Boyazoglu J, Hammond K, eds. Workshop on Developing Breeding Strategies for Lower Input Animal Production Environments, Bella, Italy, 22-25 September 1999. ICAR Technical Series No. 3: 347-354.

Dieye PN, Faye A, Seydi M, Cissé SA. 2002. Production laitière périurbaine et amélioration des revenus des petits producteurs en milieu rural au Sénégal. Cahiers Agricultures 11(4): 251-257.

Diop M, Fall A, Niang S. 1993. Mise en place d'un système génétique à noyau ouvert sur le bétail N'Dama au Sénégal - Le dépistage des vaches dans les troupeaux villageois. In: Proc. Atelier sur l'Amélioration génétique des bovins en Afrique de l'Ouest, Banjul, 17-21 Octobre 1992, pp. 269-280.

Ehui S, Barry MB, Williams TO, Koffi-Koumi M, Zeleka P. 2003. Quelles politiques pour améliorer la compétitivité des petits éleveurs dans le corridor central de l'Afrique de l'Ouest: implications pour le commerce et l'intégration régionale. In: ILRI Proceedings, ILRI, Nairobi, Kenya, 79 p.

Ejlertsen M, Poole J, Marshall K. 2013. Traditional breeding objectives and practices of goat, sheep and cattle smallholders in The Gambia and implications in relation to the design of breeding interventions. Tropical Animal Health and Production 45(1): 219 229. DOI: 10.1007/s11250-012-0194-1.

Fall A. 1989. An alternative selection scheme for N'Dama cattle in Senegal. No. 030. Kolda (Sénégal) : CRZ.

Fall A, Diop M, Sandford J, Wissocq YJ, Trail JC. 1982. Évaluation des productivités des ovins Djallonke et des taurins N'Dama au centre de recherches zootechniques de Kolda, Sénégal. ILCA Research Report 3. Addis Ababa (Ethiopia): ILCA (International Livestock Centre for Africa), 74 p.

Haile A, Gizaw S, Getachew T, Mueller JP, Amer P, Rekik M, et al. 2019. Community-based breeding programmes are a viable solution for Ethiopian small ruminant genetic improvement but require public and private investments. J Anim Breed Genet 136: 319-328. DOI: 10.1111/jbg.1240.

ILRI. 2010. Gestion durable du bétail ruminant endemique en Afrique de l'Ouest: étude de référence, Sénégal. Nairobi (Kenya): ILRI.

Jaitner J, Corr N, Dempfle L. 2003. Ownership pattern and management practices of cattle herds in the Gambia: Implications for a breeding programme. Tropical Animal Health and Production 35(2): 179-187.

Mueller JP, Rischkowsky B, Haile A, Philipsson J, Mwai O, Besbes B, et al. 2015. Community-based livestock breeding programmes: Essentials and examples. Journal of Animal Breeding and Genetics 132(2): 155-168. DOI: 10.1111/jbg. 12136.

Naessens J. 2006. Bovine trypanotolerance: A natural ability to prevent severe anaemia and haemophagocytic syndrome? International Journal for Parasitology 36: 521-528.

Planchenault D, Tall SH, Traoré MT. 1984. Amélioration génétique des bovins N'Dama. Études en milieu extensif au Mali. I. Caractéristiques du bétail N'Dama au ranch de Madina-Diassa Croissance, Barymétrie. Rev Elev Med Vet Pays Trop 37: 488-495.

Sissokho MM, Diop M, Niang S. 1993. Mise en place d'un système de sélection à noyau ouvert pour l'amélioration génétique du 
taurin Ndama: résultats du «screening» des vaches exceptionnelles dans le département de Kolda (Sénégal). Rapport d'avancement. Kolda: ISRA/CRZ, 17 p.

Tamboura T, Bibe B, Babile R. 1982. Résultats expérimentaux sur le croisement entre races locales et races laitières améliorées au Mali. Rev Elev Med Vet Pays Trop 35: 401-412.

Touré SM. 1977. La trypanotolérance. Revue de connaissances. Rev Elev Med Vet Pays Trop 30: 157-174.
Traoré SA, Reiber C, Zárate AV. 2018. Productive and economic performance of endemic N'Dama cattle in southern Mali compared to Fulani Zebu and their crossbreds. Livest Sci 209: 77-85. DOI: 10.1016/j.livsci.2018.01.013.

Wurzinger M, Escareño L, Pastor F, Salinas H, Iñiguez L, Sölkner J. 2013. Design and implementation of a community-based breeding program for dairy goats in Northern Mexico. Tropical and Subtropical Agroecosystems 16: 289-296.

Citation de l'article : Camara Y, Sissokho MM, Sall M, Farnir F, Antoine-Moussiaux N. 2020. Programmes de sélection du bovin N'Dama en Afrique de 1'Ouest: cas du Sénégal, du Mali et de la Gambie. Cah. Agric. 29: 11. 